\title{
Identification of Nonalcoholic Fatty Liver Disease following Pancreatic Surgery in a Western Cohort Using a Novel Radiographic Technique
}

\author{
Sidney Olefson ${ }^{1}$, Melissa Jackson ${ }^{1}$, David J. Grand ${ }^{2}$, Kevin P. Charpentier ${ }^{3}$, Nirav Makwana ${ }^{2}$ \\ and Kittichai Promrat*4
}

\begin{abstract}
${ }^{1}$ Department of Internal Medicine, Warren Alpert Medical School of Brown University, Providence, RI, USA; ${ }^{2}$ Department of Diagnostic Imaging, Warren Alpert Medical School of Brown University, Providence, RI, USA; ${ }^{3}$ Department of Surgery, Warren Alpert Medical School of Brown University, Providence, RI, USA; ${ }^{4}$ Division of Gastroenterology and Hepatology, Warren Alpert Medical School of Brown University, Providence, RI, USA
\end{abstract}

\begin{abstract}
Background and Aims: While traditional risk factors for the development of nonalcoholic fatty liver disease (NAFLD) relate to metabolic syndrome, several Asian studies have suggested a high rate of de novo NAFLD following pancreaticoduodenectomy (PD). The aim of this study is to identify de novo NAFLD after pancreatic surgery and its associated risk factors. Methods: A retrospective cohort of patients at a single center that underwent PD or distal pancreatectomy (DP) over 7 years was identified. Pre- and postoperative contrast-enhanced computed tomography scans of the abdomen were reviewed, including attenuation measurements of the liver, spleen, and muscle. Primary outcomes included hepatic attenuation, liver to muscle ratio (LMR), and liver to spleen ratio (LSR). Results: Of the 96 patients (mean age 64.3) included, $70 \%$ underwent PD, and $30 \%$ underwent DP. The mean LMR decreased significantly from 1.81 to 1.66 $(p=0.02)$, noted only in men. No interaction effect with LMR was observed with surgical type, chemotherapy, blood loss, pancreatic enzyme replacement, or transaminases. LMR decreased in $55 \%$ of subjects. Conclusions: Increased fatty infiltration, as evidence by decreased LMR, was found among men that underwent PD and DP within a year of surgery. This may be related to weight loss and malabsorption and deserves further investigation.

(C) 2015 The Second Affiliated Hospital of Chongqing Medical University. Published by XIA \& HE Publishing Ltd. All rights reserved.
\end{abstract}

\section{Introduction}

Nonalcoholic fatty liver disease (NAFLD) is the most common liver disorder worldwide, ${ }^{1}$ and its prevalence is increasing. ${ }^{2-4}$ In the United States, NAFLD is estimated to affect $10-35 \%$ of patients, ${ }^{3}$ and biopsy-based studies show the prevalence of nonalcoholic steatohepatitis (NASH) is $3-5 \% .{ }^{2,3} \mathrm{NASH}$ may progress to cirrhosis in up to $20 \%$ of patients. ${ }^{5}$ Traditional risk factors for the development of NAFLD include elevated body mass index (odds ratio (OR) 6.6 for $>35 \mathrm{~kg} / \mathrm{m}^{2}$ ), diabetes mellitus (OR 2.4), hypertension (OR 1.6), hypercholesterolemia (OR 1.3), and sedentary lifestyle (OR 1.3). ${ }^{2}$

Risk factors associated with metabolic syndrome are thought to play a role in the pathophysiology of NAFLD via insulin resistance, which leads to increased lipolysis from white adipose tissue and increased hepatic fat accumulation. ${ }^{1}$ Several Asian-based studies report the development of hepatic steatosis following pancreaticoduodenectomy (PD), ${ }^{6-9}$ which typically lacks traditional risk factors for NAFLD.

$P D$, often used for the treatment of periampullary disease, involves en bloc resection of the distal stomach, duodenum, head of the pancreas, and proximal jejunum. ${ }^{10}$ Improvement in the surgical technique and approach has been associated with a tremendous decline in mortality to as low as $0.8 \% .{ }^{11,12}$ Increased postoperative longevity has highlighted previously unknown consequences of the significant alteration in internal anatomy, including gastric stasis and delayed transit, gastric ulcers, pancreatic exocrine, and endocrine dysfunction. ${ }^{13,14}$

Although previous studies based in Japan have identified intraoperative blood loss, ${ }^{6}$ exocrine insufficiency, ${ }^{7}$ and pancreatic adenocarcinoma ${ }^{15}$ as factors associated with the development of NAFLD postoperatively, this phenomenon has not been studied in a North American population. Furthermore, it has not been evaluated in patients undergoing isolated resection of the pancreas, which is the organ implicated in the development of NAFLD after PD. The purpose of this study is to evaluate de novo NAFLD after pancreatic surgery and to identify its associated risk factors.

\section{Methods}

\section{Overview}

This retrospective review was conducted at a single site (Rhode Island Hospital, USA) and was approved by the Lifespan 
Olefson S. et al: NAFLD following pancreatic surgery

Institutional Review Board (IRB). Study patients were identified and included if they underwent PD or distal pancreatectomy (DP) between 1 January 2007 and 31 December 2013. Patients were retrospectively followed from 3 to 12 months following the procedure for both radiographic and laboratory data. A comprehensive chart review was conducted to elicit age, gender, surgery type, use of adjuvant chemotherapy and pancreatic enzyme replacement, laboratory values, comorbidities, and body mass index (BMI) before and after surgery. Patients were excluded if there was a documented history of alcohol abuse, viral hepatitis (either documented or the presence of a positive viral serology), or if relevant and timely imaging studies could not be located.

\section{Radiology}

Pre- and postoperative intravenous (IV), contrast-enhanced computed tomography (CT) scans of the abdomen were reviewed in conjunction with a radiologist. Preoperative images were obtained within 3 months of surgery, and postoperative images obtained between 3 and 12 months after surgery were included. All CT scans were performed at a single institution with the type and amount of IV contrast material and scan delay held constant. Using manually drawn regions of interest on the portal venous phase images, the attenuation (measured in Hounsfield units, or HUs) of three areas of the liver, one of the spleen, and one in the paraspinal muscles were measured for each patient on both pre- and postoperative studies (Fig. 3, Fig. 4). The paraspinal muscles were included to account for the high proportion of DP patients that underwent splenectomy at the time of surgery. Ratios were calculated using the average liver attenuation divided by the organ of interest (spleen or muscle). Liver biopsies were not available to confirm pathologic diagnosis of hepatic steatosis. Because of the lack of standardization in the literature using liver to muscle ratio (LMR) with contrast-enhanced $C T$, we were unable to define NAFLD for individual patients, but inferred hepatic deposition based on a decrease in the ratio on a population level.

\section{Statistics}

Data were analyzed using SAS Software 9.4 (SAS Inc., USA). The LMR was examined by comparing the pre- vs. postoperative periods, DP vs. PD, BMI, and male vs. female using mixed linear modeling with sandwich estimation, where observations were nested within subjects. Mixed modeling was also used to examine the relationship between the pre- and postoperative periods, LMR, and DP vs. PD on aspartate aminotransferase (AST), alanine aminotransferase (ALT), alkaline phosphatase, and total bilirubin (TB). Pearson correlations were used to examine the relationship between blood loss and LMR. Differences in various demographics and biomarkers between DP and PD were examined both pre- and postoperatively using t-tests. Multiple comparisons were examined using Bonferroni corrections. Alpha was set at the 0.05 level, and all interval estimates were calculated for $95 \%$ confidence.

\section{Results}

There were 204 patients who underwent pancreatic surgery during the study period. Of these, 108 were excluded due to lack of imaging $(n=97)$, history of alcoholism $(n=6)$, viral
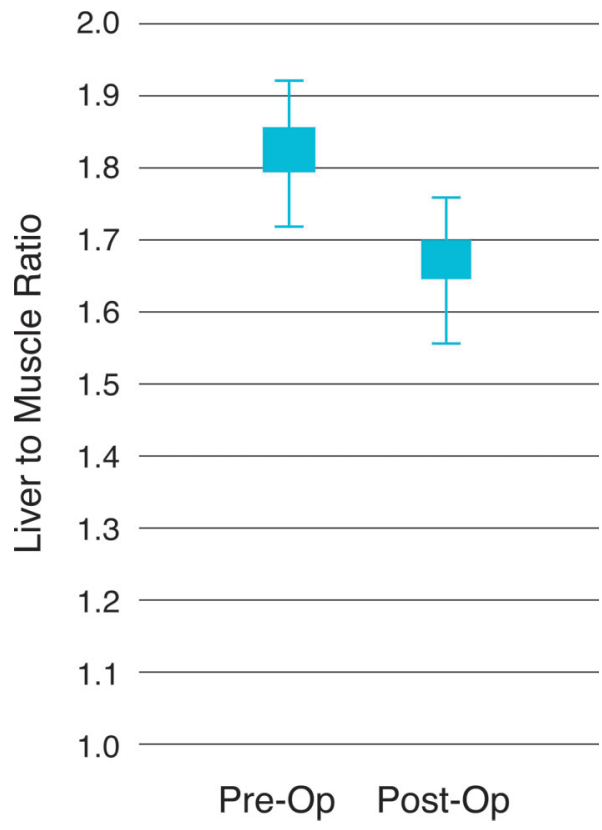

Fig. 1. Liver to muscle ratio in entire cohort.

hepatitis $(n=3)$, and hepatic metastases $(n=2)$. Lack of imaging occurred when a different modality (such as magnetic resonance imaging (MRI)) was used or the patient had outside imaging unable to be located. Of the 96 remaining patients, $67(70 \%)$ underwent PD, and 29 (30\%) underwent DP. The overall mean duration of days of follow-up for the postoperative imaging was 287.5 days (95\% confidence interval (CI), 226.1-348.8 days). Demographics, including age, sex, pre- and postoperative BMI, surgical blood loss, use of adjuvant chemotherapy, and postoperative pancreatic enzyme supplementation, are shown in Table 1. Patients that underwent DP were younger than the PD group (Table 1). The DP group, however, had more adjuvant chemotherapy and higher rates of pancreatic enzyme supplementation documented than the PD group.

Hemoglobin was significantly decreased in the PD group but not in the DP group, and this difference persisted between

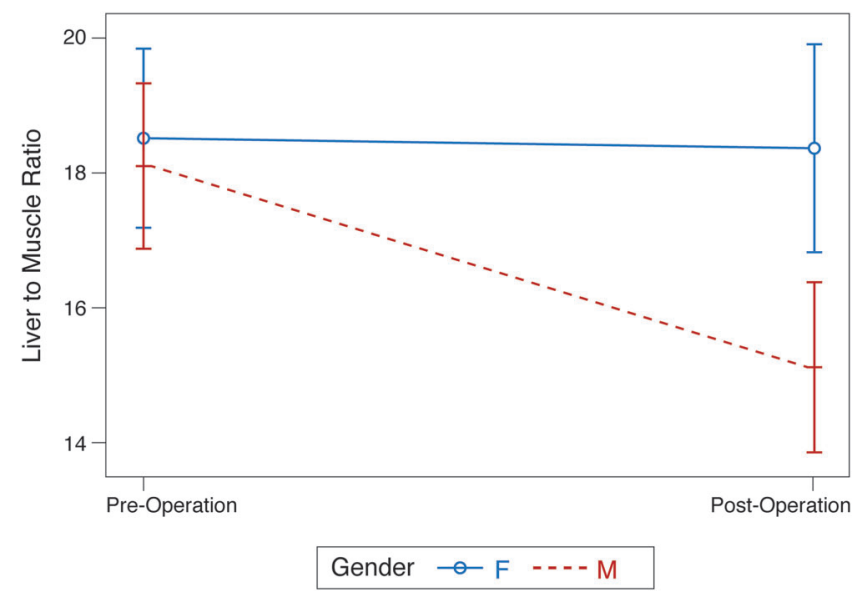

Fig. 2. Liver to muscle ratio by gender. 


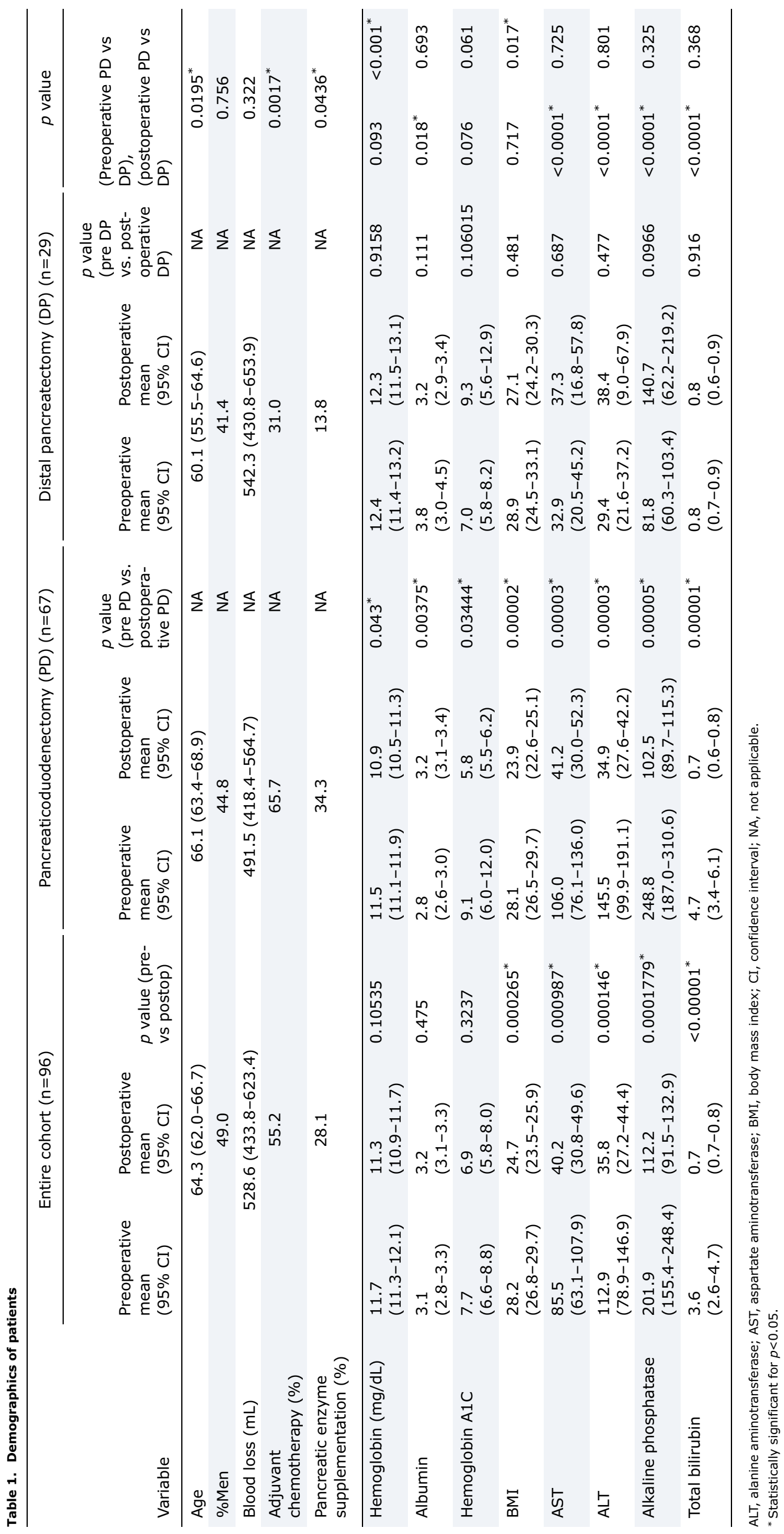


PD and DP during the postoperative period. Other significant decreases that occurred in the PD group include BMI, hemoglobin A1c, AST, ALT, alkaline phosphatase, and TB. Albumin was significantly increased in the PD group, and this preoperative difference was noted between the PD and DP groups (Table 1).

There was a significant decrease $(p=0.0255)$ in LMR between the pre- and postoperative periods, from 1.81 preoperatively to 1.66 postoperatively, an $8.5 \%$ decrease (Fig. 1 , Table 3). This was seen in 53 of 96 patients (55\%). There was an interaction effect with BMI for LMR reduction that approached significance $(p=0.10)$. Notably, this effect was more pronounced among PD patients than patients that underwent DP (Table 1).

There were notable sex differences observed in the LMR (Fig. 2). Females had higher LMRs (1.83, 95\% CI [1.68-1.99]) than males $(1.50,95 \%$ CI $[1.37-1.63])$, postoperatively $(p=0.01)$. Although the LMR did not change for women between the pre- and postoperative periods $(p=0.99)$, the LMR was significantly reduced for men from 1.81 (95\% CI [1.63-1.93]) to 1.50 (95\% CI [1.37-1.63]) $(p=0.003)$.

Pathology of resected specimens are included in Table 2. While pancreatic adenocarcinoma (60\%) and ampullary carcinoma (13\%) dominated the PD group, the DP group was dominated by pancreatic adenoma $(20 \%)$, neuroendocrine tumor $(24 \%)$, and serous cystadenoma (17\%).

Radiographic characteristics of all included groups are included in Table 3. Differences were not noted between the pre- and postoperative periods for liver attenuation or liver to spleen ratio (LSR). A significant decrease in LMR was observed in the overall cohort but not in either group when analyzed separately.

There was no significant effect of pre- and postoperative periods and surgery type on LMR $(p=0.59)$. The presence of malignancy, as determined by pathology, did not significantly influence LMR postoperatively $(p=0.96)$ nor did the use of chemotherapy (when divided in 5-fluorouracil based therapy, gemcitabine based therapy, and no chemotherapy). There was no interaction observed between surgical groups, cancer, and the preoperative and postoperative periods $(p=0.82)$. There was also no interaction between $\Delta \mathrm{LMR}$ and surgical group with AST, ALT, alkaline phosphatase, and TB (all $p>0.05$ ).

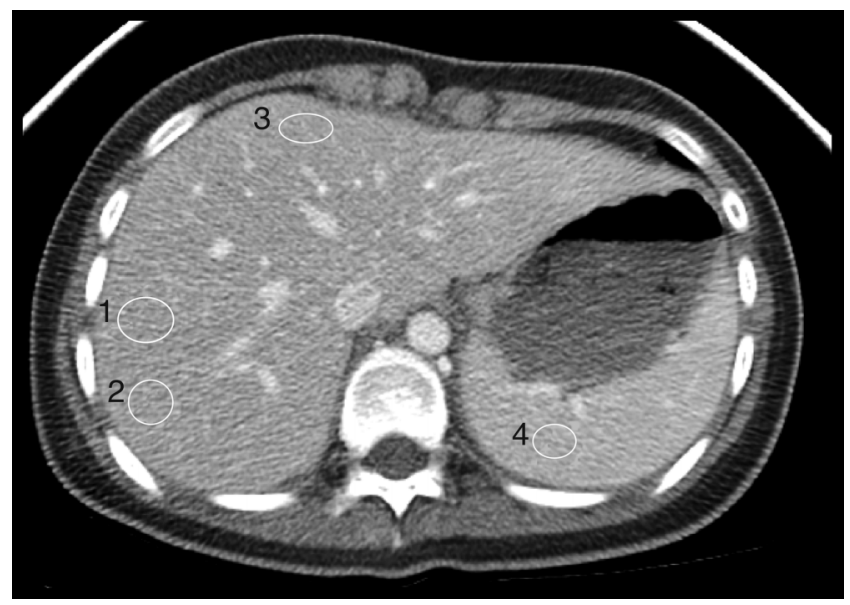

Fig. 3. Areas of interest for attenuation measurement.

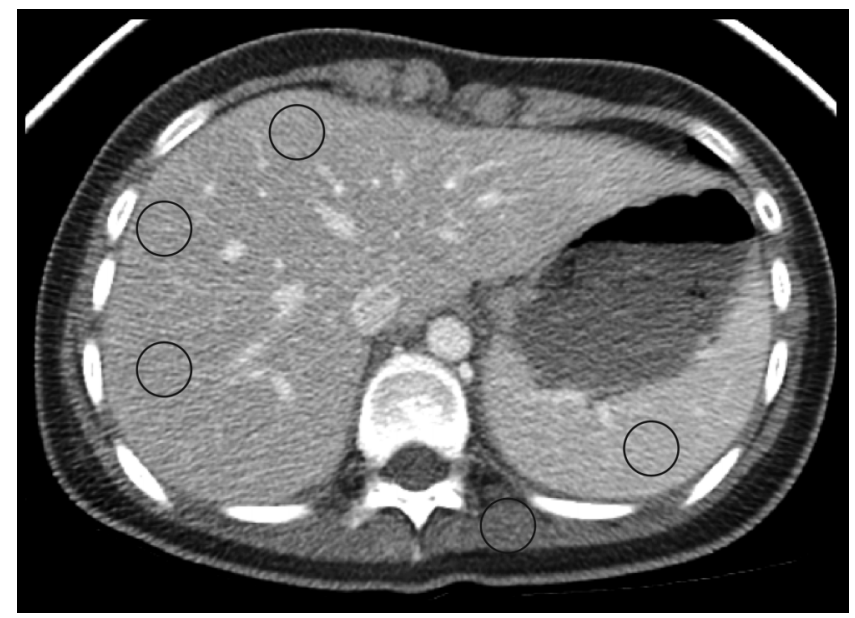

Fig. 4. Measured diameters of areas of interest for attenuation.

No correlation was observed between amount of blood loss and LMR ( $r=-0.159)$ as an aggregate. This held true for PD $(r=-0.08)$ and DP $(r=-0.185)$ when analyzed separately. Furthermore, use of postoperative pancreatic enzymes did not correlate with LMR as an aggregate $(p=0.45)$ or by surgical type $(p=0.98)$.

\section{Discussion}

In this study, we found that NAFLD developed in male patients after PD and DP, as determined by a reduction in hepatic attenuation compared with muscle (LMR). This reduction in attenuation was observed in greater than half $(55 \%)$ of the study patients. These findings corroborate those of other studies with Asian subjects that observed this phenomenon in $12-37 \%$ of subjects. ${ }^{6,7,15,16}$ Post-PD reductions in hepatic attenuation ranged from 10 to $36 \mathrm{HU} \cdot{ }^{6,7,15}$ We found this both in patients that underwent PD and DP, a population not included in the previous studies.

We employed two novel radiographic techniques to determine and test for the presence of postoperative hepatic steatosis. First, we used contrast-enhanced CT, the predominant source of radiographic surveillance at our center. Previous studies using noncontrast CT have shown an LSR of 0.9 to be a specific marker of hepatic steatosis. $6,8,11,16$ Some authors ${ }^{17}$ have suggested comparable specificity with contrast-enhanced CT, specifically in the portal phase, but with low sensitivity. ${ }^{18}$ These studies utilized the spleen for reference. Although type and amount of contrast material injected, injection timing, and scan parameters may influence $\mathrm{LSR}^{19}$ these variables were held constant across scans.

Due to the high prevalence of patients that underwent splenectomy in our study, we used muscle as a comparator to detect change in hepatic attenuation. This technique was reported and validated by previous studies ${ }^{20,21}$ and was shown to have high inter and intrareader reproducibility. ${ }^{20}$

Here, there was no interaction between LMR and cancer diagnosis, use of chemotherapy, blood loss, use of postoperative pancreatic enzyme replacement, or alteration in liver enzymes. Previous studies had found that the presence of pancreatic adenocarcinoma, ${ }^{15}$ perioperative blood loss, ${ }^{6,7}$ and exocrine insufficiency ${ }^{7}$ affected the course of hepatic steatosis development after PD. While our study found no interaction 


\begin{tabular}{|c|c|c|c|}
\hline Pathology & $\begin{array}{l}\text { Entire cohort } \\
\mathrm{N}(\%)\end{array}$ & $\begin{array}{l}\text { Pancreaticoduodenectomy } \\
(n=67) N(\% \text { of group) }\end{array}$ & $\begin{array}{l}\text { Distal pancreatectomy } \\
(n=29) N(\% \text { of group) }\end{array}$ \\
\hline Pancreatic adenocarcinoma & $46(47.9)$ & $40(59.7)$ & $6(20.1)$ \\
\hline Pancreatic neuroendocrine tumor & $11(11.4)$ & $4(6.0)$ & $7(24.1)$ \\
\hline Intra-papillary mucinous neoplasm (IPMN) & $11(11.4)$ & $7(10.4)$ & $4(13.8)$ \\
\hline Ampullary/periampullary carcinoma & $9(9.4)$ & $9(13.4)$ & $0(0.0)$ \\
\hline Mucinous cystadenoma & $1(1.0)$ & $0(0.0)$ & $1(3.4)$ \\
\hline Serous cystadenoma & $5(5.2)$ & $0(0.0)$ & $5(17.2)$ \\
\hline Duodenal adenocarcinoma & $2(2)$ & $2(3.0)$ & $0(0.0)$ \\
\hline Autoimmune pancreatitis & $1(1.0)$ & $0(0.0)$ & $1(3.4)$ \\
\hline $\begin{array}{l}\text { Pancreatic adenocarcinoma with neuroendocrine } \\
\text { differentiation }\end{array}$ & $1(1.0)$ & $0(0.0)$ & $1(3.4)$ \\
\hline Cholangiocarcinoma & $1(1.0)$ & $1(1.5)$ & $0(0.0)$ \\
\hline Gastric adenocarcinoma & $1(1.0)$ & $0(0.0)$ & $1(3.4)$ \\
\hline Liposarcoma & $1(1.0)$ & $1(1.5)$ & $0(0.0)$ \\
\hline Metastatic malignancy (from renal cell carcinoma) & $1(1.0)$ & $0(0.0)$ & $1(3.4)$ \\
\hline Chronic pancreatitis & $1(1.0)$ & $0(0.0)$ & $1(3.4)$ \\
\hline Schwannoma & $1(1.0)$ & $1(1.5)$ & $0(0.0)$ \\
\hline Solid pseudotumor papillary neoplasm & $1(1.0)$ & $0(0.0)$ & $1(3.4)$ \\
\hline Gastrointestinal stromal tumor & $1(1.0)$ & $1(1.5)$ & $0(0.0)$ \\
\hline Retention cyst & $1(1.0)$ & $1(1.5)$ & $0(0.0)$ \\
\hline
\end{tabular}

between decreased LMR and blood loss, there was a significant decline in postoperative hemoglobin (Table 1). Previous authors $^{6}$ have postulated that intraoperative blood loss induces ischemia with resultant insulin resistance. However, we cannot conclude that long-term postoperative anemia is due to intraoperative loss. The inability to detect an interaction of LMR with cancer and chemotherapy does not exclude the possibility of a potential interaction effect. However, the observation of a postoperative decrease in LMR, seen in both surgical groups, suggests that fatty infiltration may be related to pancreatic volume loss.

Prior studies have found that the use of postoperative pancreatic enzyme replacement is protective against the development of NAFLD. ${ }^{7,8,15,16}$ Failure to find a protective effect with postoperative use of pancreatic enzymes does not exclude the possibility that it may ameliorate NAFLD. First, we were unable to obtain data on the diagnosis of pancreatic insufficiency. Second, we were unable to identify dosing. Nakagawa et al. postulated that inadequate dosing may not be able to counter the effects of weight loss and malnutrition. ${ }^{7}$

BMI and sex were associated with a decrease in LMR. While the interaction of BMI with postoperative LMR approached statistical significance, there was a significant reduction in BMI of the study cohort from 28.5 at baseline to $25.5 \mathrm{~kg} / \mathrm{m}^{2}$ postoperatively. This trend, replicated in other studies, $7,8,16$ suggested that the pathophysiology associated with the development of NAFLD mimics that of fatty infiltration in malnutrition. In the absence of metabolic syndrome and insulin resistance, malnourishment may lead to diminished lipoprotein synthesis, leading to decreased exportation of lipids from the liver. ${ }^{8}$ Tanaka et al. suggested that a decrease in apolipoprotein B synthesis, which assists in very low density lipoprotein secretion from hepatocytes, may also play a role. ${ }^{16}$ However, our study did show a statistically significant increase in hemoglobin A1C for patients that underwent DP rather than PD (Table 1). Some have found that endocrine dysfunction correlated with postoperative hepatic steatosis following PD, ${ }^{15}$ while others have not. ${ }^{7}$

Furthermore, we found a significant interaction of LMR with sex, where fat deposition was notably increased in men. This finding is consistent with prior epidemiologic studies, which have shown that the incidence of NAFLD is higher in men, even when adjusting for intra-abdominal adiposity. ${ }^{22}$ Some have theorized that estrogen is protective against adipogenesis $^{23}$ This hypothesis was corroborated by others who have noted that the presence of NAFLD is greater among postmenopausal women than premenopausal women. ${ }^{24}$

The decrease in LMR in our study was not correlated with an elevation in liver enzymes. There were only preoperative differences between the two surgical groups (Table 1) for AST, ALT, alkaline phosphatase, and TB. A decrease in transaminases occurred most predominantly in the PD group between the pre- and postoperative period. We believe this effect was largely related to the relief of biliary obstruction due to the cholestatic pattern of elevation, which was relieved by surgical reconstruction and was observed previously. ${ }^{25}$

While elevations in transaminases remain the most common biochemical abnormality in patients with NAFLD, ${ }^{26}$ it is not a specific marker. A wide spectrum of histologic findings has been noted among patients with normal ALT 


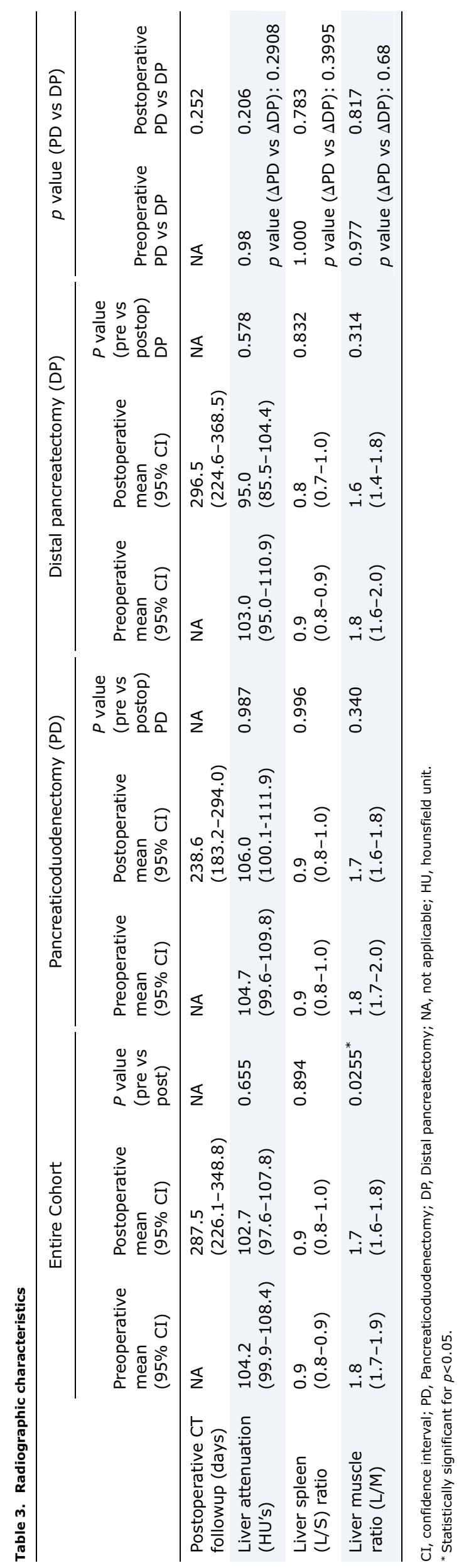


values. ${ }^{27,28}$ One study ${ }^{8}$ noted that AST was significantly elevated among post-PD patients compared with controls, however, this difference was small and still within the range of normal (37 compared with $25 \mathrm{IU} / \mathrm{L}$ ).

Our study had several limitations. As noted, the use of LMR, while used by previous investigators, is not a goldstandard for detecting hepatic steatosis. Our intention, however, was not to detect or quantify baseline steatosis but to measure change in hepatic fat content within the same patient before and after surgery. With scan parameters kept constant, a decrease in liver parenchymal attenuation can only be attributed to hepatic fat deposition. We, therefore, concluded that a decrease in LMR implied fatty infiltration. Unfortunately, lack of standardized ranges for this measurement did not allow us to determine which specific patients met criteria for de novo NAFLD. Thus, we were unable to select a specific threshold by which to classify patients with and without NAFLD. Furthermore, the sex discrepancy noted in our study may be influenced by inherent sex differences in muscle composition. While this possible baseline difference might affect the absolute value of muscle attenuation, it should not change the pre- and postoperative images and, therefore, should not affect comparison of LMRs. The finding of diminished LMR was replicated in both surgical groups. Although this study was retrospective and some patients lacked sufficient follow-up, our sample size was greater than those in previously reported studies ${ }^{15,16}$ and incorporated patients that underwent isolated pancreatic resection.

\section{Conclusions}

Our study demonstrates that male patients are at high risk for developing hepatic steatosis in the year following pancreatic surgery, based on decreased LMR as determined by contrastCT studies. Although the pathogenesis of this phenomenon is not well understood, it may be intrinsic to the removal of a portion of the pancreas itself, a side-effect of alternate digestive anatomy or a combination of both. Furthermore, the use of serum transaminases does not appear sensitive enough to detect subtle changes of fatty composition in this setting. Future prospective studies ought to delineate this phenomenon using more specific quantitative metrics of hepatic fat, such as MRI and to gain further insight into alternate pathways for fatty liver pathogenesis.

\section{Conflict of interest}

None.

\section{Author contributions}

Study concept and design (SO, MJ, KP, DJG), acquisition of the data (SO, MJ, DJG, NM), drafting the manuscript (SO, MJ), critical revision of the manuscript (KPC, DJG, KP), statistical analysis (SO), study supervision (KP).

\section{References}

[1] Asrih M, Jornayvaz FR. Metabolic syndrome and nonalcoholic fatty liver disease: Is insulin resistance the link? Mol Cell Endocrinol 2015 December. doi: $10.1016 /$ j.mce.2015.02.018
[2] Lazo M, Hernaez R, Eberhardt MS, Bonekamp S, Kamel I, Guallar E, et al. Prevalence of nonalcoholic fatty liver disease in the United States: the Third National Health and Nutrition Examination Survey, 1988-1994. Am J Epidemiol 2013;178:38-45. doi: 10.1093/aje/kws448.

[3] Vernon G, Baranova A, Younossi ZM. Systematic review: the epidemiology and natural history of non-alcoholic fatty liver disease and non-alcoholic steatohepatitis in adults. Aliment Pharmacol Ther 2011;34:274-285. doi: 10. 1111/j.1365-2036.2011.04724.x.

[4] Younossi ZM, Stepanova M, Afendy M, Fang Y, Younossi Y, Mir H, et al. Changes in the prevalence of the most common causes of chronic liver diseases in the United States from 1988 to 2008. Clin Gastroenterol Hepatol 2011;9:524-530, e1; quiz e60. doi: 10.1016/j.cgh.2011.03.020.

[5] Matteoni CA, Younossi ZM, Gramlich T, Boparai N, Liu YC, McCullough AJ. Nonalcoholic fatty liver disease: a spectrum of clinical and pathological severity. Gastroenterology 1999;116:1413-1419. doi: 10.1016/S00165085(99)70506-8.

[6] Ito Y, Kenmochi T, Shibutani S, Egawa T, Hayashi S, Nagashima A, et al. Evaluation of predictive factors in patients with nonalcoholic fatty liver disease after pancreaticoduodenectomy. Am Surg 2014;80:500-504.

[7] Nakagawa N, Murakami Y, Uemura K, Sudo T, Hashimoto Y, Kondo N, et al. Nonalcoholic fatty liver disease after pancreatoduodenectomy is closely associated with postoperative pancreatic exocrine insufficiency. J Surg Oncol 2014;110:720-726. doi: 10.1002/jso.23693.

[8] Nomura R, Ishizaki Y, Suzuki K, Kawasaki S. Development of hepatic steatosis after pancreatoduodenectomy. AJR Am J Roentgenol 2007;189: 1484-1488. doi: 10.2214/AJR.07.2809.

[9] Sim EH, Kwon JH, Kim SY, Jung SM, Maeng LS, Jang JW, et al. Severe steatohepatitis with hepatic decompensation resulting from malnutrition after pancreaticoduodenectomy. Clin Mol Hepatol 2012;18:404-410. doi: 10.33 50/cmh.2012.18.4.404.

[10] Yu HH, Shan YS, Lin PW. Effect of pancreaticoduodenectomy on the course of hepatic steatosis. World J Surg 2010;34:2122-2127. doi: 10.1007/s00268010-0636-8.

[11] Fernandez-del Castillo C, Morales-Oyarvide V, McGrath D, Wargo JA, Ferrone $\mathrm{CR}$, Thayer SP, et al. Evolution of the Whipple procedure at the Massachusetts General Hospital. Surgery 2012;152:S56-S63. doi: 10.1016/j.surg.2012. 05.022.

[12] Saraee A, Vahedian-Ardakani J, Saraee E, Pakzad R, Wadji MB. Whipple procedure: a review of a 7-year clinical experience in a referral center for hepatobiliary and pancreas diseases. World J Surg Oncol 2015;13:98. doi: 10.1186/s12957-015-0523-8.

[13] Huang J], Yeo CJ, Sohn TA, Lillemoe KD, Sauter PK, Coleman J, et al. Quality of life and outcomes after pancreaticoduodenectomy. Ann Surg 2000;231: 890-898.

[14] Ohtsuka T, Tanaka M, Miyazaki K. Gastrointestinal function and quality of life after pylorus-preserving pancreatoduodenectomy. J Hepatobiliary Pancreat Surg 2006;13:218-224. doi: 10.1007/s00534-005-1067-z.

[15] Kato H, Isaji S, Azumi Y, Kishiwada M, Hamada T, Mizuno S, et al. Development of nonalcoholic fatty liver disease (NAFLD) and nonalcoholic steatohepatitis (NASH) after pancreaticoduodenectomy: proposal of a postoperative NAFLD scoring system. J Hepatobiliary Pancreat Sci 2010;17:296-304. doi: 10.1007/s00534-009-0187-2.

[16] Tanaka N, Horiuchi A, Yokoyama T, Kaneko G, Horigome N, Yamaura T, et al. Clinical characteristics of de novo nonalcoholic fatty liver disease following pancreaticoduodenectomy. J Gastroenterol 2011;46:758-768. doi: 10.100 7/s00535-011-0370-5.

[17] Kim DY, Park SH, Lee SS, Kim HJ, Kim SY, Kim MY, et al. Contrast-enhanced computed tomography for the diagnosis of fatty liver: prospective study with same-day biopsy used as the reference standard. Eur Radiol 2010;20: 359-366. doi: 10.1007/s00330-009-1560-x.

[18] Lawrence DA, Oliva IB, Israel GM. Detection of hepatic steatosis on contrastenhanced CT images: diagnostic accuracy of identification of areas of presumed focal fatty sparing. AJR Am J Roentgenol 2012;199:44-47. doi: 10. 2214/AJR.11.7838.

[19] Johnston RJ, Stamm ER, Lewin JM, Hendrick RE, Archer PG. Diagnosis of fatty infiltration of the liver on contrast enhanced CT: limitations of liver-minusspleen attenuation difference measurements. Abdom Imaging 1998;23: 409-415.

[20] Speliotes EK, Massaro JM, Hoffmann U, Foster MC, Sahani DV, Hirschhorn JN, et al. Liver fat is reproducibly measured using computed tomography in the Framingham Heart Study. J Gastroenterol Hepatol 2008;23:894-899. doi: $10.1111 / \mathrm{j} .1440-1746.2008 .05420 . x$.

[21] Panicek DM, GiessCS, SchwartzLH. Qualitative assessment of liver for fatty infiltration on contrast-enhanced CT: is muscle a better standard of reference than spleen? J Comput Assist Tomogr 1997;21:699-705.

[22] Gilsanz V, Chung SA, Kaplowitz N. Differential effect of gender on hepatic fat. Pediatr Radiol 2011;41:1146-1153. doi: 10.1007/s00247-011-2021-7.

[23] Cooke PS, Naaz A. Role of estrogens in adipocyte development and function. Exp Biol Med (Maywood) 2004;229:1127-1135. 
[24] Geer EB, Shen W. Gender differences in insulin resistance, body composition, and energy balance. Gend Med 2009;1:60-75. doi: 10.1016/j.genm.2009. 02.002 .

[25] Hughes C, Hurtuk MG, Rychlik K, Shoup M, Aranha GV. Preoperative liver function tests and hemoglobin will predict complications following pancreaticoduodenectomy. J Gastrointest Surg 2008;12:1822-1827; discussion 1827-1829. doi: 10.1007/s11605-008-0680-y.

[26] McCullough AJ. The clinical features, diagnosis and natural history of nonalcoholic fatty liver disease. Clin Liver Dis 2004;8:521-533, viii.
[27] Mofrad $P$, Contos $M J$, Haque $M$, Sargeant $C$, Fisher RA, Luketic VA, et al. Clinical and histologic spectrum of nonalcoholic fatty liver disease associated with normal ALT values. Hepatology 2003;37:1286-1292. doi: 10 . 1053/jhep.2003.50229.

[28] Wong VW, Wong GL, Tsang SW, Hui AY, Chan AW, Choi PC, et al. Metabolic and histological features of non-alcoholic fatty liver disease patients with different serum alanine aminotransferase levels. Aliment Pharmacol Ther 2009;29:387-396. doi: 10.1111/j.1365-2036.2008. 03896.x. 